\title{
Discontinuities of the Chemical Potential in Reduced Density Matrix Functional Theory
}

By N. N. Lathiotakis ${ }^{1}$, S. Sharma ${ }^{2,6,7, *}$, N. Helbig ${ }^{4,6}$, J. K. Dewhurst ${ }^{2,3,6}$, M. A. L. Marques ${ }^{5,6}$, F. Eich ${ }^{2,3,6}$, T. Baldsiefen ${ }^{2,6}$, A. Zacarias ${ }^{2,6}$, and E. K. U. Gross $2,6,7, * *$

1 Theoretical and Physical Chemistry Institute, National Hellenic Research Foundation, Vass. Constantinou 48, 11635 Athens, Greece

${ }^{2}$ Institut für Theoretische Physik, Freie Universität Berlin, Arnimallee 14, 14195 Berlin, Germany

${ }^{3}$ Fritz Haber Institute of the Max Planck Society, Faradayweg 4-6, 14195 Berlin, Germany

${ }^{4}$ Nano-Bio Spectroscopy group, Dpto. Física de Materiales, Universidad del País Vasco, Centro de Física de Materiales CSIC-UPV/EHU-MPC and DIPC, Av. Tolosa 72, E-20018 San Sebastián, Spain

${ }^{5}$ LPMCN, Université Claude Bernard Lyon I and CNRS, F-69622 Villeurbanne, France.

${ }^{6}$ European Theoretical Spectroscopy Facility

${ }^{7}$ Max-Planck-Institut für Mikrostrukturphysik, Weinberg 2, 06120 Halle, Germany.

(Received July 5, 2009; accepted October 19, 2009)

\section{Discontinuities / Chemical Potential / Reduced Density Matrix Functional Theory}

Using the discontinuity of the chemical potential as a function of excess charge, the fundamental gaps for finite systems and the band gaps of extended solids are determined within reduced density matrix functional theory. We also present the necessary and sufficient conditions for the one-body reduced density matrix of a system with fractional charge to be ensemble $\mathrm{N}$ representable. The performance of most modern day reduced density matrix functionals is assessed for the gaps and the correlation energy of finite systems. Our results show that for finite systems the $\mathrm{PNOF}, \mathrm{BBC} 3$, and power functionals yield very accurate correlation energies while for a correct description of the fundamental gap the removal of self-interaction terms is essential. For extended solids we find that the power functional captures the correct band gap behavior for conventional semiconductors as well as strongly correlated Mott insulators, where a gap is obtained in absence of any magnetic ordering.

\footnotetext{
* Corresponding author. E-mail: nsas@zedat.fu-berlin.de

**CCorresponding author. E-mail: hardy@mpi-halle.de
} 


\section{Introduction}

Reduced density matrix functional theory (RDMFT) has recently appeared as an alternative approach to handle complex systems. It has shown great potential for improving upon density functional theory (DFT) results for finite [1] and extended [2] systems. As the basic variable [3,4] RDMFT uses the one-body reduced density matrix (1-RDM)

$$
\gamma\left(\mathbf{x}, \mathbf{x}^{\prime}\right)=N \int d \mathbf{x}_{2} \ldots d \mathbf{x}_{N} \Psi^{*}\left(\mathbf{x}^{\prime}, \mathbf{x}_{2}, \ldots \mathbf{x}_{N}\right) \Psi\left(\mathbf{x}, \mathbf{x}_{2}, \ldots \mathbf{x}_{N}\right),
$$

where $\Psi$ denotes the many-body wave function and $\mathbf{x}=(\mathbf{r}, \sigma)$. Diagonalization of this matrix produces a set of spinor valued natural orbitals [3], $\phi_{j}$, and occupation numbers, $n_{j}$, leading to the spectral representation

$$
\gamma\left(\mathbf{x}, \mathbf{x}^{\prime}\right)=\sum_{j=1}^{\infty} n_{j} \phi_{j}(\mathbf{x}) \phi_{j}^{*}\left(\mathbf{x}^{\prime}\right)
$$

where the necessary and sufficient conditions for ensemble $N$-representability [5] require $0 \leq n_{j} \leq 1$ for all $j$, and $\sum_{j=1}^{\infty} n_{j}=N$.

In terms of $\gamma$, the total ground state energy [4] of the interacting system is (atomic units are used throughout)

$$
\begin{aligned}
E[\gamma]= & -\frac{1}{2} \sum_{\sigma} \int \lim _{\mathbf{r} \rightarrow \mathbf{r}^{\prime}} \nabla_{\mathbf{r}}^{2} \gamma\left(\mathbf{r}, \sigma, \mathbf{r}^{\prime}, \sigma\right) d^{3} r^{\prime}+\sum_{\sigma} \int \rho(\mathbf{r}, \sigma) V_{\text {ext }}(\mathbf{r}) d^{3} r \\
& +\frac{1}{2} \sum_{\sigma \sigma^{\prime}} \int \frac{\rho(\mathbf{r}, \sigma) \rho\left(\mathbf{r}^{\prime}, \sigma^{\prime}\right)}{\left|\mathbf{r}-\mathbf{r}^{\prime}\right|} d^{3} r d^{3} r^{\prime}+E_{\mathrm{xc}}[\gamma],
\end{aligned}
$$

where $\rho(\mathbf{r}, \sigma)=\gamma(\mathbf{x}, \mathbf{x}), V_{\text {ext }}$ is a given external potential, and $E_{\mathrm{xc}}$ we call the exchange-correlation (xc) energy functional. In principle, Gilbert's [4] generalization of the Hohenberg-Kohn theorem to the 1-RDM guarantees the existence of a functional $E[\gamma]$ whose minimum yields the exact $\gamma$ and the exact ground-state energy of the systems characterized by the external potential $V_{\text {ext }}(\mathbf{r})$. In practice, however, the correlation energy is an unknown functional of the 1-RDM and needs to be approximated. Regarding these approximations, RDMFT has a twofold advantage over conventional DFT; one, the kinetic and exchange energies of the interacting system are explicit functionals of $\gamma$ and two, the extra degrees of freedom, i.e. the off-diagonal elements of $\gamma\left(\mathbf{x}, \mathbf{x}^{\prime}\right)$, make the theory amenable to a wider range of functional forms for the xc energy.

If the Hamiltonian of the system is diagonal in spin, the 1-RDM decouples in separate matrices for each spin channel. In order to describe systems with a specific spin $S_{z}$ one can further restrict the natural orbitals to be spin independent $\phi_{j}(\mathbf{x})=\varphi_{j}(\mathbf{r})$, and only allow the occupation numbers to aquire a spin-dependence [6]. Most of the commonly used approximations can then be cast in the form

$$
E_{x c}[\gamma]=-\frac{1}{2} \sum_{\sigma} \sum_{j, k=1}^{\infty} f\left(n_{j \sigma}, n_{k \sigma}\right) \iint d^{3} r d^{3} r^{\prime} \frac{\varphi_{j}(\mathbf{r}) \varphi_{j}^{*}\left(\mathbf{r}^{\prime}\right) \varphi_{k}\left(\mathbf{r}^{\prime}\right) \varphi_{k}^{*}(\mathbf{r})}{\left|\mathbf{r}-\mathbf{r}^{\prime}\right|}
$$


with various choices for the function $f$. In this work we employ the Müller [7], GU [8], BBC1-3 [9], PNOF [10, 11], CGA [12], and the power [2] functionals. Other functionals of the above type are given in Refs. [13-18]. Eq. (3) can also be modified by including a density-dependent parameter which is determined from the homogeneous electron gas resulting in the so-called $\mathrm{BBC}++$ functional [19].

Unlike DFT, RDMFT does not result in a set of single-particle Kohn-Sham like equations and the total energy in Eq. (3) has to be minimized directly with respect to the 1-RDM, or, alternatively, with respect to the occupation numbers and natural orbitals. In order to ensure the conservation of particle number as well as the orthonormality of the orbitals one introduces Lagrange multipliers and minimizes [20,21]

$$
F[\gamma]=E[\gamma]-\sum_{\sigma} \mu_{\sigma}\left(\sum_{j=1}^{\infty} n_{j \sigma}-N_{\sigma}\right)-\sum_{j, k=1}^{\infty} \epsilon_{j k}\left(\int d^{3} r \varphi_{j}(\mathbf{r}) \varphi_{k}^{*}(\mathbf{r})-\delta_{j k}\right),
$$

where $\varepsilon_{j k}=\varepsilon_{k j}^{*}$. One can show that the Lagrange multipliers $\mu_{\sigma}$ are identical to the (spin-dependent) chemical potentials $[22,23]$, i.e.

$$
\mu_{\sigma}=\frac{\partial E}{\partial N_{\sigma}} .
$$

Following the same path as in DFT [24-26] it can be proved that for the exact energy functional the chemical potential shows a discontinuity at integer particle numbers. The size of the discontinuity is identical to the fundamental gap, i.e. the difference between the ionization potential and the electron affinity, and is given as [23]

$$
\Delta=\min _{\sigma}\left(\lim _{w_{\sigma} \rightarrow 0} \mu_{\sigma}\left(N_{\sigma}+w_{\sigma}, N_{\sigma^{\prime}}\right)\right)-\max _{\sigma}\left(\lim _{w_{\sigma} \rightarrow 0} \mu_{\sigma}\left(N_{\sigma}+w_{\sigma}, N_{\sigma^{\prime}}\right)\right),
$$

where $\sigma^{\prime} \neq \sigma$. In practice the band gap is determined by calculating $\mu(w)$ for -1 $\leq w \leq 1$ and constructing tangents (for details see Section 3 and Refs. [22,23,2]). This procedure involves the minimization of the total energy with respect to the 1-RDM of a system with fractional number of particles, $\gamma^{N+w}$. To perform this minimization, the necessary and sufficient conditions for ensemble $N$-representability of $\gamma^{N+w}$ are needed. We present these conditions in Section 2. Results for finite and periodic systems are given in Sections 3.1 and 3.2, respectively, and we summarize our findings in Section 4.

\section{Ensemble N-representability conditions}

In this section we show that the necessary and sufficient ensemble $N$-representability conditions for a 1-RDM, $\gamma^{N+w}\left(\mathbf{r}, \mathbf{r}^{\prime}\right)$, with fractional particle number are

$$
\sum_{i} n_{i}=N+w \quad \text { and } \quad 0 \leq n_{i} \leq 1
$$


In order to show this, we first prove that the conditions in Eq. (8) are necessary. The 1-RDM for a fractional number of particles is defined as:

$$
\gamma^{N+w}\left(\mathbf{r}, \mathbf{r}^{\prime}\right)=w \gamma^{N+1}\left(\mathbf{r}, \mathbf{r}^{\prime}\right)+(1-w) \gamma^{N}\left(\mathbf{r}, \mathbf{r}^{\prime}\right),
$$

where $\gamma^{N}$ and $\gamma^{N+1}$ are ensemble $N$-representable $N$ and $N+1$ particle 1-RDM, respectively. Using the triangular inequality (which is valid for positive definite matrices like $\gamma$ ), from Eq. (9) we get

$$
\begin{aligned}
& \left\{n_{i}^{N+w}\right\}_{\max } \leq w\left\{n_{i}^{N+1}\right\}_{\max }+(1-w)\left\{n_{i}^{N}\right\}_{\max } \\
\Rightarrow & \left\{n_{i}^{N+w}\right\}_{\max } \leq w+(1-w)=1
\end{aligned}
$$

and

$$
\begin{aligned}
& \left\{n_{i}^{N+w}\right\}_{\min } \geq w\left\{n_{i}^{N+1}\right\}_{\min }+(1-w)\left\{n_{i}^{N}\right\}_{\min } \\
\Rightarrow & \left\{n_{i}^{N+w}\right\}_{\min } \geq 0 .
\end{aligned}
$$

Eqs. (10) and (11) clearly show that eigenvalues of any ensemble $N$-representable 1-RDM for a fractional number of particles, satisfy the conditions in Eq. (8).

As a second step, we demonstrate that the conditions in Eq. (8) are sufficient for ensemble $N$-representability of $\gamma^{N+w}$. To do this we first show that the set $G$ of all the vectors $\mathbf{x}=\left(x_{1}, x_{2} \ldots\right)$ such that $0 \leq x_{i} \leq 1$ and $\Sigma_{i} x_{i}=N+w$ is convex, with a set of extreme elements, $G_{e}$, that have $N$ components to be 1 , one component $w$ and the rest 0 . That this set is convex is obvious: let $\mathbf{x}, \mathbf{y} \in G$ then $\mathbf{z}=$ $\alpha \mathbf{x}+(1-\alpha) \mathbf{y}$ for all $0 \leq \alpha \leq 1$, must also belong to $G$.

Elements of type $\mathbf{z} \in G_{e}$ are extreme elements of this convex set $G$ which can be proved as follows, write element $\mathbf{z} \in G_{e}$ as

$$
\mathbf{z}=\alpha \mathbf{x}+(1-\alpha) \mathbf{y} \quad \forall 0 \leq \alpha \leq 1,
$$

where $\mathbf{x}, \mathbf{y} \in G$. Element-wise, with elements of $\mathbf{z}$ arranged in descending order, one obtains the following set of equations

$$
\alpha x_{i}+(1-\alpha) y_{i}= \begin{cases}1, & 1 \leq i \leq N \\ w, & i=N+1 \\ 0, & i>N+1\end{cases}
$$

The first and last relations in Eq. (13) imply

$$
\begin{aligned}
& x_{i}=y_{i}=1, \quad 1 \leq i \leq N \\
& x_{i}=y_{i}=0, \quad i>N+1 .
\end{aligned}
$$

and since $\mathbf{x}, \mathbf{y} \in G$ their components have the property $\Sigma_{i} x_{i}=\Sigma_{i} y_{i}=N+w$, which in turn means $x_{i}=y_{i}=w$ when $i=N+1$ and so $\mathbf{z}=\mathbf{x}=\mathbf{y}$ and hence $\mathbf{z}$ is extreme by definition. 
We also need to show that no other element of $G$ is extreme. This is done by showing any element $\mathbf{x} \in G \backslash G_{e}$ can be written as $\mathbf{x}=\alpha \mathbf{y}+(1-\alpha) \mathbf{z}$ for all 0 $\leq \alpha \leq 1$. Let $\mathbf{x}$ have $M$ non-zero components such that $M>N+1$. We can do a slight rearrangement and write elements of $\mathbf{x}$ such that $x_{1}>x_{2}>x_{3}>\ldots>x_{M}$, this ensures $0<x_{M} \leq x_{M-1}<1$. One can now choose $\varepsilon>0$ such that $\varepsilon=$ $\min \left(x_{M}, x_{M-1}, 1-x_{M}, 1-x_{M-1}\right)$ and with this construct two vectors $\mathbf{y} \neq \mathbf{z} \in G$ with

$$
\begin{aligned}
& \mathbf{y}=\left(x_{1}, x_{2}, \ldots, x_{M-1}+\epsilon, x_{M}-\epsilon\right) \\
& \mathbf{z}=\left(x_{1}, x_{2}, \ldots, x_{M-1}-\epsilon, x_{M}+\epsilon\right)
\end{aligned}
$$

such that $\mathbf{x}=1 / 2(\mathbf{y}+\mathbf{z})$. This demonstrates that any element of $G \backslash G_{e}$ cannot be extreme.

With this in hand any matrix $A\left(\mathbf{r}, \mathbf{r}^{\prime}\right)$ satisfying conditions in Eq. (8) can be written as

$$
A\left(\mathbf{r}, \mathbf{r}^{\prime}\right)=\sum_{i}\{\mathbf{x}\}_{i} \phi_{i}(\mathbf{r}) \phi_{i}^{*}\left(\mathbf{r}^{\prime}\right)=\sum_{i}\left\{\sum_{j} \alpha_{j} z^{j}\right\}_{i} \phi_{i}(\mathbf{r}) \phi_{i}^{*}\left(\mathbf{r}^{\prime}\right)
$$

where $\mathbf{x} \in G$ and $\mathbf{z} \in G_{e}$. Rearranging the terms, Eq. (16) reads

$$
\begin{aligned}
A\left(\mathbf{r}, \mathbf{r}^{\prime}\right) & =\alpha_{1} \sum_{i} z_{i}^{1} \phi_{i}(\mathbf{r}) \phi_{i}^{*}\left(\mathbf{r}^{\prime}\right)+\alpha_{2} \sum_{i} z_{i}^{2} \phi_{i}(\mathbf{r}) \phi_{i}^{*}\left(\mathbf{r}^{\prime}\right)+\ldots \\
& =\alpha_{1}\left[\sum_{i}^{N} \phi_{i}(\mathbf{r}) \phi_{i}^{*}\left(\mathbf{r}^{\prime}\right)+w \phi_{N+1}(\mathbf{r}) \phi_{N+1}^{*}\left(\mathbf{r}^{\prime}\right)\right]+\ldots \\
& =\alpha_{1}\left[(1-w) \gamma_{1}^{N}+w \gamma_{1}^{N+1}\right]+\alpha_{2}\left[(1-w) \gamma_{2}^{N}+w \gamma_{2}^{N+1}\right]+\ldots \\
& =(1-w)\left[\sum_{j} \alpha_{j} \gamma_{j}^{N}\right]+w\left[\sum_{j} \alpha_{j} \gamma_{j}^{N+1}\right]
\end{aligned}
$$

The terms in the square brackets in Eq. (17) are $N$ and $N+1$ particle 1-RDM [5] thus making the left hand side of Eq. (17) ensemble $N$-representable.

\section{The fundamental gap}

\subsection{Finite systems}

In this section, we study the behavior of 1-RDM functionals for fractional total number of electrons for finite systems. As explained, the discontinuity of the chemical potential at integer particle number is a direct measure of the fundamental gap for the exact theory [22,23]. It is of great importance to examine if present day functionals reproduce this behavior.

The LiH molecule will serve as a test case for closed-shell systems. In Fig. 1 we show the chemical potential $\mu$ as a function of the fractional total number of particles $M$ around $M=4$, the particle number of the neutral system. As we see, 
there are functionals (GU, PNOF, power) that reproduce a step-like behavior in close resemblance to the discontinuity of the exact theory. Functionals that do not involve the removal of self-interaction (SI) terms, i.e. Müller, the BBC's as well as $\mathrm{BBC}++$, do not produce any discontinuity. We also observe that for the $\mathrm{GU}$, and PNOF functionals the size of the discontinuity is in agreement with the fundamental gap of $\mathrm{LiH}$ (see Table 1). For the power functional, a discontinuity appears for $\alpha>0.6$ but it is very small at first and increases with increasing $\alpha$. At the same time the cancellation of SI terms between the Hartree and the xc energies improves with increasing $\alpha$ thus confirming the importance of their removal for a correct description of the fundamental gap. One has to choose values close to Hartree-Fock $(\alpha=1)$ in order for the discontinuity to be comparable to the experimental gap. Unfortunately, the SI is a serious problem in the application of the GU and PNOF functionals for the band gap of periodic systems, since in the case of extended electronic states (Bloch-states) the SI terms are zero. A way-around [22] is to assume that the natural orbitals are localized Wannier-like states in which case the SI is non-zero. However, this imposes an extra restriction on the optimal 1-RDM, and we are essentially using a different functional. As was shown in Ref. [2] this limitation does not apply to the power functional.

The results shown in Fig. 1 were obtained by distributing the additional charge equally over the two spin channels, i.e. the system remained closed-shell. One can, however, restrict this charge to one spin channel at a time. This has more physical meaning, since, in the limit of a whole extra electron, the spin of the ground state of the system is altered. Adding an extra electron to the $\mathrm{LiH} \mathrm{mo-}$ lecule, for example, leads to a spin-doublet state [6,23]. If that process is done incrementally, the extra charge should go into the same spin in order to reach the spin doublet. In that way, there are two chemical potentials, $\mu^{\uparrow}, \mu^{\downarrow}$, one for each spin, and they can be functions of both $M^{\uparrow}$ and $M^{\downarrow}$ [6,23]. Keeping either $M^{\uparrow}$ or $M^{\downarrow}$ constant and equal to the neutral system value, $M^{\uparrow}=N^{\uparrow}$ or $M^{\downarrow}=$ $N^{\downarrow}$, we obtain 4 different chemical potentials, namely, $\mu^{\uparrow}\left(M^{\uparrow}, N^{\downarrow}\right), \mu^{\uparrow}\left(N^{\uparrow}, M^{\downarrow}\right)$, $\mu^{\downarrow}\left(N^{\uparrow}, M^{\downarrow}\right)$, and $\mu^{\downarrow}\left(M^{\uparrow}, N^{\downarrow}\right)$.

In Fig. 2 we show $\mu^{\uparrow}\left(M^{\uparrow}, N^{\downarrow}\right)$ and $\mu^{\downarrow}\left(M^{\uparrow}, N^{\downarrow}\right)$ for the open-shell treatment of the LiH molecule. As we see, only $\mu^{\uparrow}\left(M^{\uparrow}, N^{\downarrow}\right)$ shows a discontinuous behavior while $\mu^{\downarrow}\left(M^{\uparrow}, N^{\downarrow}\right)$ is perfectly continuous. We did not include the chemical potentials for a fractional number of particles in the down channel since those are identical to their spin-up counterparts for closed-shell neutral systems. Comparing the shape and size of discontinuities for open- and closed-shell treatments, Figs. 1 and 2, we note that i) they are of similar size, both being good measures of the fundamental gap, ii) the open-shell treatment resembles the true discontinuity better, being almost horizontal far from the step region.

If the neutral system is open-shell we have two functions that are discontinuous, $\mu^{\uparrow}\left(M^{\uparrow}, N^{\downarrow}\right)$ and $\mu^{\downarrow}\left(N^{\uparrow}, M^{\downarrow}\right)$ which are no longer equal. Although this might seem to complicate things the identification of the gap follows a physical principle: we have to pick up the smallest possible discontinuity [23] and that value is 


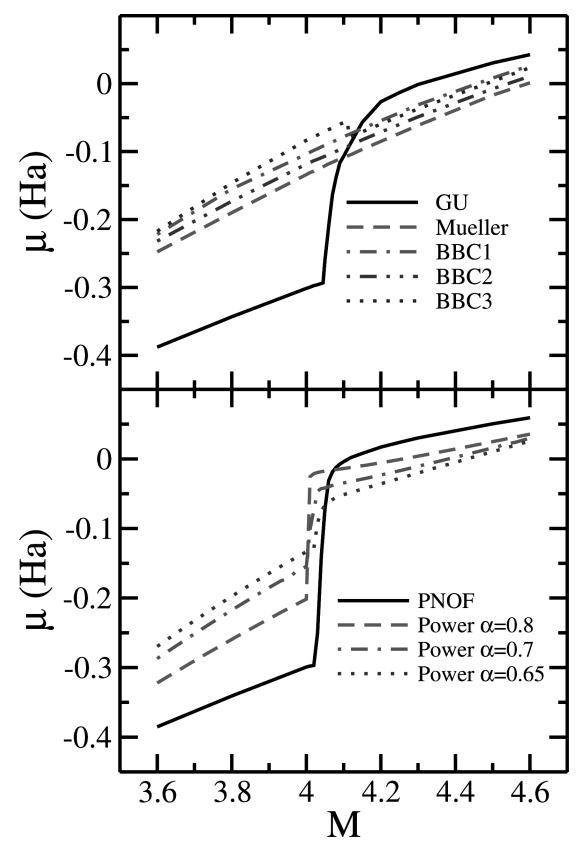

Fig. 1. The function $\mu(M)$ for the $\mathrm{LiH}$ molecule using the closed-shell treatment for the Goedecker-Umrigar, the Müller, BBC1, BBC2, BBC3, PNOF, and power functionals. The GU and the PNOF involve a complete removal of the SI terms and reproduce a pronounced step in resemblance of the discontinuity of the exact theory. For the power functional the degree of SI removal depends on the value of $\alpha$ which is directly reflected in the size of the step for different $\alpha$.

the measure of the fundamental gap. The physical meaning behind this procedure is that we have to select the horizontal branches of the chemical potentials that correspond to the energetically favorable spin state when electrons are added to/ removed from the system. (In the following we take the majority spin to be spin up.)

In Fig. 3, we show $\mu^{\downarrow}\left(N^{\uparrow}, M^{\downarrow}\right)$ and $\mu^{\uparrow}\left(M^{\uparrow}, N^{\downarrow}\right)$ for the Li atom doublet. In this case we have to select $\mu^{\uparrow}\left(M^{\uparrow}, N^{\downarrow}\right)$ for negative $w$ and $\mu^{\downarrow}\left(N^{\uparrow}, M^{\downarrow}\right)$ for positive $w$. This choice reflects the fact that it is energetically favorable to remove an electron from the majority spin channel while the addition of an electron favours the minority spin. In Fig. 4, we include the same plots for the $\mathrm{Na}$ and $\mathrm{F}$ atoms. The case of $\mathrm{Na}$ is quite similar to $\mathrm{Li}$ while for $\mathrm{F}$, we get the gap from the function $\mu^{\downarrow}\left(N^{\uparrow}, M^{\downarrow}\right)$ only. This means that, for $\mathrm{F}$, it is favorable to add an electron to and remove an electron from the minority spin yielding the positive ion triplet state.

In Table 1, we give the results obtained by backwards extrapolation of the chemical potential functions to extract numerical values for the gaps. Although 


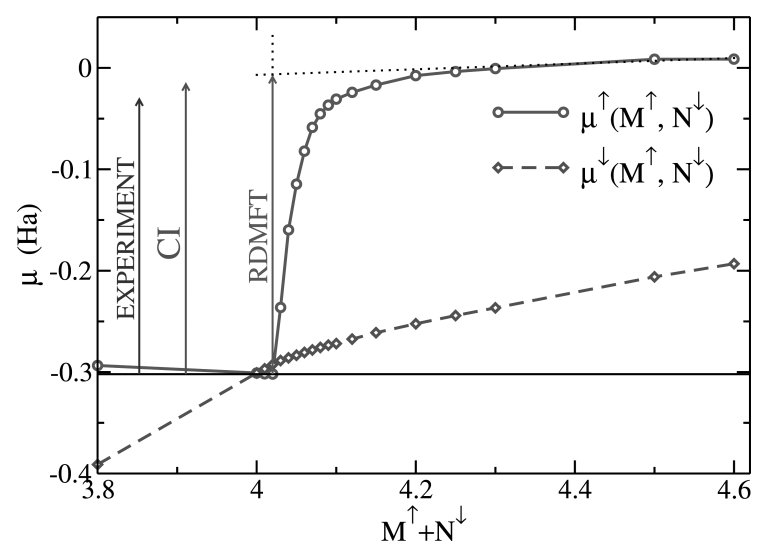

Fig. 2. Chemical potentials as functions of fractional electron numbers $M$ for the $\mathrm{LiH}$ molecule using the GU functional in an open-shell treatment. $N^{\downarrow}$ is kept constant and equal to the integer, neutral-system value, $N^{\downarrow}=2$. For comparison, the experimental and CI values of the fundamental gap are included.

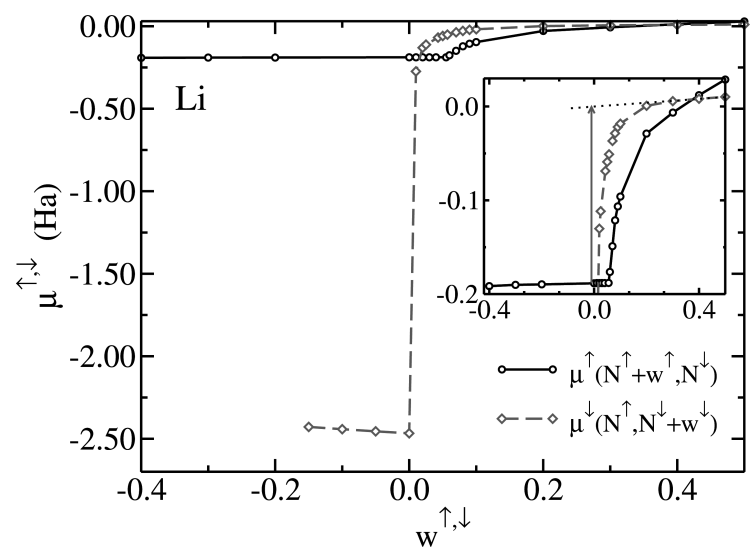

Fig. 3. The chemical potentials $\mu^{\sigma}$ of an electron fraction $w^{\sigma}$ added (subtracted) to the neutral $\mathrm{Li}$ atom. In the inset, we show the region where the value of the fundamental gap is extracted from as the difference of the upper level of $\mu^{\downarrow}\left(N^{\uparrow}, M^{\downarrow}\right)$ and the lower level of $\mu^{\uparrow}\left(M^{\uparrow}, N^{\downarrow}\right)$.

this procedure has a certain degree of ambiguity, one can see that the extracted values agree very well with experiment and other theoretical calculations. For finite systems, one can also calculate the gap as the difference of the ionization potential and the electron affinity that are obtained from the total energy calculations for the $N, N+1$, and $N-1$ particle systems. The values for the gap obtained in this way are also given in Table 1 for comparison. One should keep in mind 

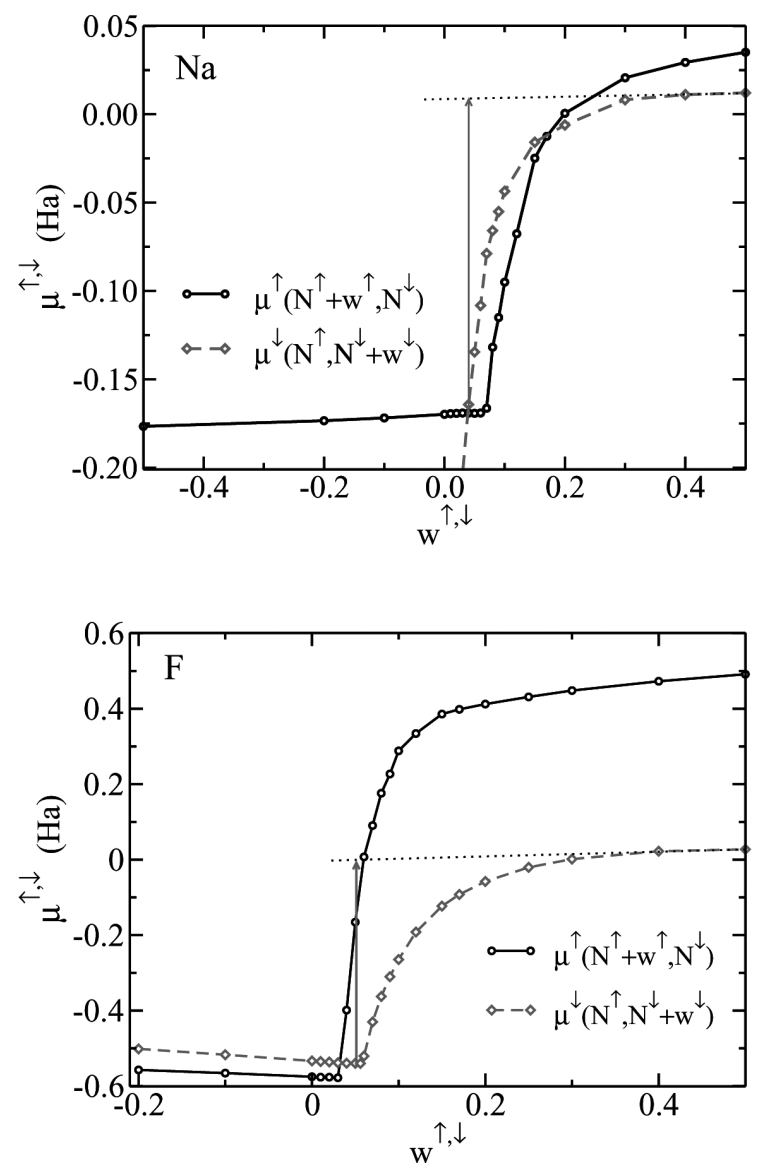

Fig. 4. Chemical potentials, $\mu^{\sigma}$, as a function of an electron fraction $w^{\sigma}$ added (subtracted) to the neutral system for the $\mathrm{Na}$ and $\mathrm{F}$ atoms.

that, for solid state systems, this direct procedure is not applicable because the addition or the removal of a single electron in an infinite solid is meaningless.

In order to design new functionals and/or improve upon the existing ones it is of great importance to know the limitations of the available approximate functionals $[1,15,33-35]$. As far as the calculation of the fundamental gaps is concerned we have seen that functionals which have their SI terms removed are far superior to functionals that contain self-interaction. However, another very important quantity to assess the quality of a functional is the correlation energy (the exchange part of $E_{x c}$ is an explicit functional of $\gamma$ ). Therefore, we have tested the performance of several approximations for a set of 148 finite systems, known as the G2/97 molecule set [36,37]. The set contains 119 neutral systems and 29 radicals, 35 nonhydrogen systems, 22 hydrocarbons, 47 substituted hydro- 
Table 1. The prediction for the fundamental gap for several atoms and small molecules using the size of the step of $\mu(M)$, and a direct calculation through the difference of the ionization potential (IP) from the electron affinity (EA) for the GU functional compared with experimental and other theoretical values. For the IP and EA we calculated the positive and negative ions with GU functional. ${ }^{a}$ QCI from Ref. [27]; ${ }^{b}$ from Ref. [28]; ${ }^{c}$ Ionization potential from [27], electron affinity from [29]; ${ }^{\mathrm{d}}$ Closed-shell treatment; ${ }^{\mathrm{e}}$ Open-shell treatment; ${ }^{\mathrm{f}}$ CISD using the same basis set as in RDMFT; ${ }^{\mathrm{g}}$ Ionization potential from [30], electron affinity from [31].

\begin{tabular}{lllll}
\hline System & $\begin{array}{l}\text { RDMFT } \\
\mu(M) \text { step }\end{array}$ & $\begin{array}{l}\text { RDMFT } \\
\text { IP-EA }\end{array}$ & $\begin{array}{l}\text { Other } \\
\text { theoretical }\end{array}$ & Experiment \\
\hline $\mathrm{Li}$ & 0.18 & 0.202 & $0.175^{\mathrm{a}}$ & $0.175^{\mathrm{b}}$ \\
$\mathrm{Na}$ & 0.18 & 0.198 & $0.169^{\mathrm{c}}$ & $0.169^{\mathrm{b}}$ \\
$\mathrm{F}$ & 0.54 & 0.549 & & $0.514^{\mathrm{b}}$ \\
$\mathrm{LiH}$ & $0.27^{\mathrm{d}}, 0.29^{\mathrm{e}}$ & 0.271 & $0.286^{\mathrm{f}}$ & $0.271^{\mathrm{g}}$ \\
\hline
\end{tabular}

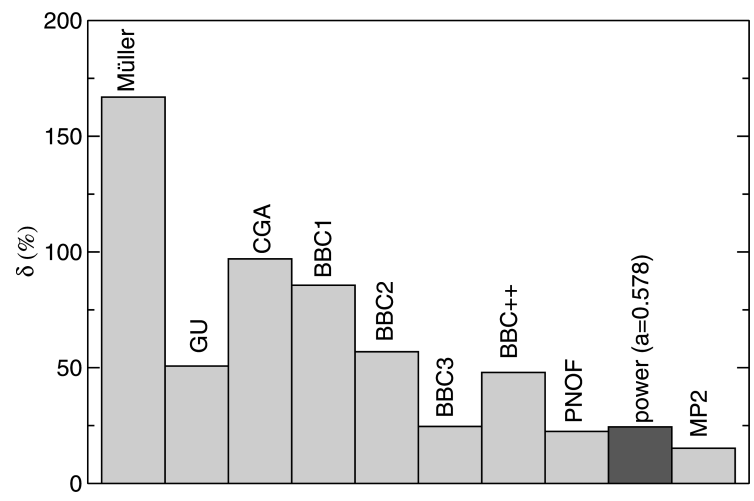

Fig. 5. Percentage deviation of the correlation energy, obtained using various 1-RDM functionals, from the exact $\operatorname{CCSD}(\mathrm{T})$ results.

carbons, and 15 inorganic hydrides. In Fig. 5, we show the relative error, $\delta$ in the correlation energy given by

$$
\delta=100 \sqrt{\sum_{G 2 \text { set }}\left[\left(E_{c}-E_{c}^{(\mathrm{ref})}\right) / E_{c}^{(\mathrm{ref})}\right]^{2} / N_{\mathrm{mol}}},
$$

where $E_{c}$ is the correlation energy obtained with the method under consideration, $E_{c}^{\text {(ref) }}$ the reference correlation energy which is obtained with the $\operatorname{CCSD}(\mathrm{T})$ method [32], and $N_{\text {mol }}$ the number of systems included in the calculation. It is apparent from Fig. 5 that the $\mathrm{BBC} 3$, the PNOF, and the power functional, with $\alpha=0.578$, yield very accurate correlation energies of finite systems (with PNOF functional being slightly better) [1]. However, for this value of $\alpha$ the gap is actually still zero while the GU functional which gave very good results for the fundamental gap performs significantly worse than other functionals for the correlation energy. It seems only the PNOF is of very high quality in both cases. 


\subsection{Periodic systems}

A dramatic failure of most density functional approximations like the local density (LDA) or generalized gradient approximations (GGA) is the incorrect prediction of a metallic state for systems that are well known experimentally as highly correlated Mott insulators. Transition metal oxides (TMO) are prime examples of this. For some of them, like $\mathrm{NiO}$ and $\mathrm{MnO}$, spin polarized calculations predict a very small band gap as a result of the anti-ferromagnetic ordering. In a spin unpolarized calculation, however, these systems are predicted to be metallic. On the other hand, these oxides are found experimentally to be insulators even above the Néel temperature [38,39]. In other words, long range magnetic order is not the reason for their insulating behavior and a real challenge for any ab-initio theory is to predict the insulating behavior for TMO in the absence of any spin ordering.

At this point it is also important to mention that periodic systems are fundamentally different from finite systems in that the addition of a finite amount of charge per unit cell to extended systems corresponds to an infinite number of electrons added to the solid as a whole (In order to maintain a finite energy density a compensating background charge is needed in addition). Thus the chemical potential of an insulating solid has a discontinuity at charge neutrality, with a slope, not necessarily zero, on either side corresponding to the continuum of states [2].

The calculations for solids are performed using the state-of-the-art full potential linearized augmented plane wave (FP-LAPW) method [40] implemented in the Elk code [41]. In Fig. 6, we show the dependence of the chemical potential on the excess charge per unit cell for 3 different solids; a prototype metal $(\mathrm{Cu})$, semi-conductor $(\mathrm{Si})$ and Mott insulator $(\mathrm{NiO})$. For $\mathrm{Cu}$ one observes a nearly linear behaviour with no sign of discontinuity which is indicative of a metallic behaviour. On the other hand, for $\mathrm{Si}$ and $\mathrm{NiO}$ the behavior is qualitatively different with the curvature of the chemical potential changing sign with addition of excess charge. We interpret this as a sign of smoothed discontinuity [2], and an estimation of the fundamental gap for these systems can be made by constructing the two tangents $[2,22,23]$ as shown in Fig. 6. In Fig. 7 are shown the percentage deviation of the calculated gap from the experimental data. Concentrating first on band insulators the agreement of the calculated gap with experiments is excellent, with an average deviation of the calculated gap being $5.5 \%$. It must be stressed that this good agreement of band gaps is found for a wide range of semiconductors/insulators, with gap ranging from $1 \mathrm{eV}(\mathrm{Ge})$ to $14.2 \mathrm{eV}(\mathrm{LiF})$ and the character of the material changing from predominantly covalent to ionic. In the case of TMO, the origin of the gap is profoundly different from conventional semiconductors/insulators. Here the gap is opened by strong Mott-Hubbard correlations. Gap formation by strong correlations is largely unaffected by the magnetic state, and so to focus exclusively on Mott-Hubbard physics we calculate these materials in the non-magnetic state. The power functional correctly 


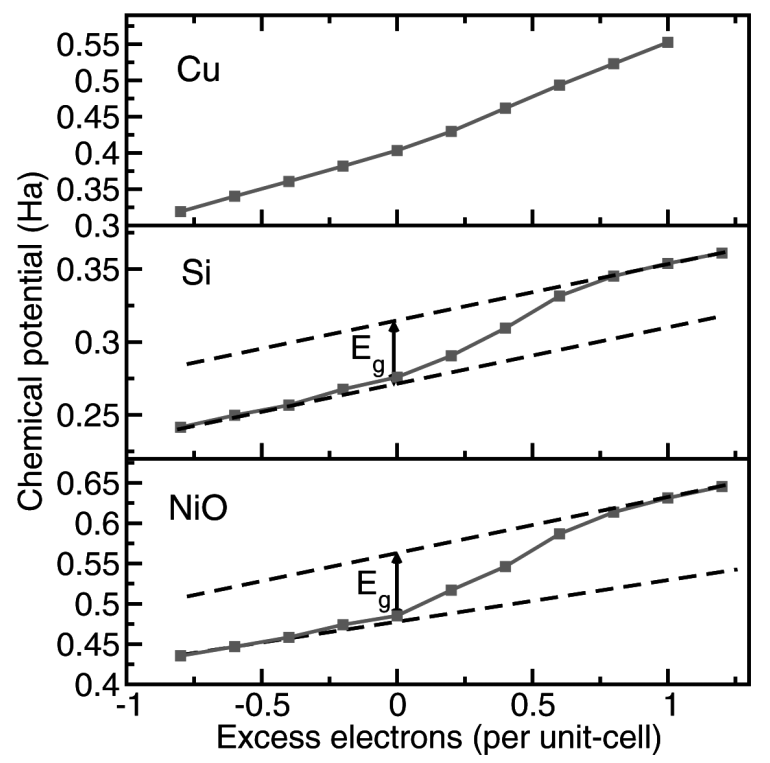

Fig. 6. Chemical potential (in $\mathrm{Ha}$ ) versus the excess charge per unit-cell for (a) $\mathrm{Cu}$, (b) $\mathrm{Si}$ and (2) $\mathrm{NiO}$. The results are obtained using the power functional with $\alpha=0.65$.

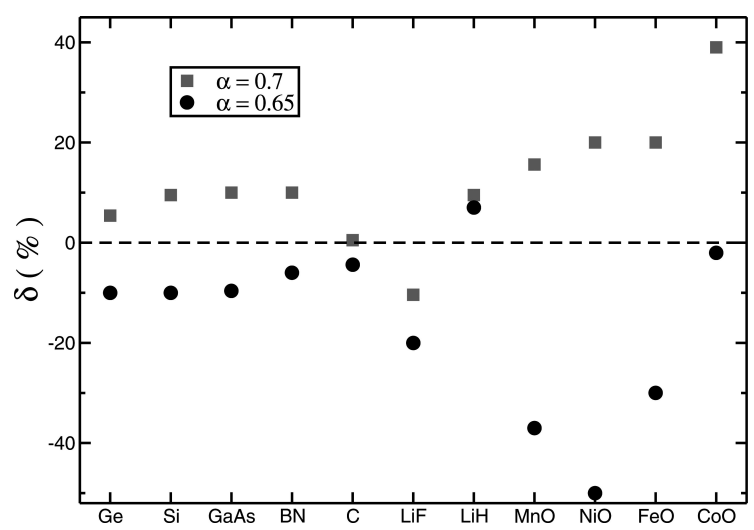

Fig. 7. Percentage deviation $(\delta)$ of calculated band gap from experiment for the power functional. Results for two different values of the exponent parameter $\alpha$ are shown.

obtains an insulating behaviour for TMO in absence of any spin-ordering. On scanning the value of $\alpha$ from 0.5 to 0.9 we find that values between 0.65 and 0.7 are optimal for a wide set of materials [2]. However, for TMO the value of gap is much more sensitive to the choice of $\alpha$ compared to the band insulators. 
At this point it is also worth mentioning that most of the band insulators and all the TMO have zero gap if the Müller functional is used.

\section{Summary}

We have used several recently proposed RDMFT functionals to assess their quality for finite and periodic systems. Our results show that the PNOF, BBC3, and power functionals perform very well for the correlation energy of finite systems. By using the discontinuity of the chemical potential, we have also determined the fundamental gaps of finite systems and found the removal of the SI terms to be of great importance. Most interestingly, the power functional also describes strong Mott-Hubbard correlations very well, as calculations of the non-magnetic TMO show. No other ab-initio approach has ever been able to achieve this. However, the optimal value of $\alpha$ is different for all three cases. The correlation energy of small atoms and molecules is best described with $\alpha=0.578$ which, however, yields a zero gap. In extended solids a value of $\alpha=0.6-0.7$ shows the best performance. The design of an ab-initio determination of $\alpha$ remains an important project for the future.

The encouraging results of the present work open up many future possibilities, such as the study of High $\mathrm{T}_{\mathrm{C}}$ superconductors in their under-doped Mott insulating phase. It should also, hopefully, stimulate efforts to further improve approximate functionals and to develop the theory formally. The latter could include an extension to magnetic or temperature dependent RDMFT as well as a method to produce quasi particle spectra.

\section{References}

1. N. N. Lathiotakis, S. Sharma and E. Gross, Phys. Rev. A 79 (2009) 040501.

2. S. Sharma, J. K. Dewhurst and E. K. U. Gross, Phys. Rev. B 78 (2008) 201103.

3. P. O. Lödwin, Phys. Rev. 97 (1955) 1974.

4. T. L. Gilbert, Phys. Rev. B 12 (1975) 2111.

5. A. Coleman, Rev. Mod. Phys. 35 (1963) 668.

6. N. N. Lathiotakis, N. Helbig, and E. K. U. Gross, Phys. Rev. A 72 (2005) 030501.

7. A. M. K. Müller, Phys. Lett. 105A (1984) 446.

8. S. Goedecker and C. J. Umrigar, Phys. Rev. Lett. 81 (1998) 866.

9. O. Gritsenko, K. Pernal, and E. J. Baerends, J. Chem. Phys. 122 (2005) 204102.

10. M. Piris, Int. J. Quant. Chem 106 (2006) 1093.

11. P. Leiva and M. Piris, J. Chem. Phys. 123 (2005) 214102.

12. G. Csányi, S. Goedecker and T. A. Arias, Phys. Rev. A 65 (2002) 032510.

13. M. A. L. Marques and N. N. Lathiotakis, Phys. Rev. A 77 (2008) 032509.

14. D. R. Rohr, K. Pernal, O. V. Gritsenko, and E. J. Baerends, J. Chem. Phys. 129 (2008) 164105.

15. N. N. Lathiotakis and M. A. L. Marques, J. Chem. Phys. 128 (2008) 184103.

16. J. Cioslowski and K. Pernal, Phys. Rev. B 71 (2005) 113103.

17. J. Cioslowski, K. Pernal, and M. Buchowiecki, J. Chem. Phys. 119 (2003) 6443.

18. K. Pernal and J. Cioslowski, J. Chem. Phys. 120 (2004) 5987. 
19. N. N. Lathiotakis, N. Helbig, A. Zacarias and E. K. U. Gross, J. Chem. Phys. 130 (2009) 064109.

20. A. J. Cohen and E. J. Baerends, Chem. Phys. Lett. 364 (2002) 409.

21. S. Goedecker and C. J. Umrigar, Phys. Rev. A 55 (1997) 1765.

22. N. Helbig, N. N. Lathiotakis, M. Albrecht and E. K. U. Gross, Europhys. Lett. 77 (2007) 67003.

23. N. Helbig, N. N. Lathiotakis and E. K. U. Gross, Phys. Rev. A 79 (2009) 022504.

24. L. J. Sham and M. Schlüter, Phys. Rev. Lett. 51 (1983) 1888.

25. L. J. Sham and M. Schlüter, Phys. Rev. B 32 (1985) 3883.

26. W. Kohn, Phys. Rev. B 33 (1986) 4331.

27. J. A. Montgomery, Jr., J. W. Ochterski and G. A. Petersson, J. Chem. Phys. 101 (1994) 5900.

28. A. A. Radzig and B. M. Smirnov, Reference Data on Atoms and Molecules. Springer Verlag, Berlin (1985).

29. J. J. D. Groote and M. Masili, J. Chem. Phys. 120 (2004) 2767.

30. H. R. Ihle and C. H. Wu, J. Chem. Phys. 63 (1975) 1605.

31. S. B. Sharp and G. I. Gellene, J. Chem. Phys. 113 (2000) 6122.

32. J. A. Pople, M. Head-Gordon and K. Raghavachari, J. Chem. Phys. 87 (1987) 5968.

33. P. Leiva and M. Piris, Int. J. Quant. Chem. 107 (2007) 1.

34. P. Leiva and M. Piris, J. Theo. Comp. Chem. 4 (2005) 1165.

35. K. Pernal and J. Cioslowski, Chem. Phys. Lett. 412 (2005) 71.

36. L. A. Curtiss, K. Raghavachari, P. C. Redfern and J. A. Pople, J. Chem. Phys. 106 (1997) 1063.

37. L. A. Curtiss, P. C. Redfern, K. Raghavachari and J. A. Pople, J. Chem. Phys. 109 (1998) 42.

38. O. Tjernberg, S. Söderholm, G. Chiaia, R. Girard, U. O. Karlsson, H. Nylén and I. Lindau, Phys. Rev. B 54 (1996) 10245.

39. W. Jauch and M. Reehuis, Phys. Rev. B 70 (2004) 195121.

40. D. J. Singh, Planewaves Pseudopotentials and the LAPW Method. Kluwer Academic Publishers, Boston (1994).

41. http://elk.sourceforge.net (2004). 